\title{
Treatment of Enteric Fever
}

\author{
Amit Arjyal ${ }^{1}$, Anil Pandit ${ }^{2}$ \\ ${ }^{1}$ Kathmandu, Nepal \\ ${ }^{2}$ Department of Internal Medicine, University of Utah Health Sciences Center, University of Utah, Salt Lake City, UT, USA 84132
}

\begin{abstract}
Enteric fever is systemic illness caused by Salmonella Typhi and Salmonella Paratyphi A, B and C. It is believed to be a readily treatable illness by many clinicians in the developing world where it is endemic; however, with the emergence of drug resistance to fluoroquinolones, treatment is becoming increasingly difficult. While drugs such as cefixime, previously believed to be effective, have been proven otherwise, new agents such as gatifloxacin and azithromycin have proven to be promising. Re-emergence of chloramphenicol sensitive strains in previously resistant areas points towards the concept of antibiotic recycling, preserving the use of older antibiotics. Antibiotic recycling has been used successfully in hospital settings. However, its usefulness in community settings, where the main burden of enteric fever resides, is challenging to manage due to logistics and a lack of infrastructure. Nalidixic acid resistance used to be a marker for clinical response to flouroquinolones; however, recent studies highlight the importance of decreased ciprofloxacin susceptibility as a better marker. Enteric fever, as a public health problem, has been tackled by protection of food and water supplies in the industrialised countries of the world. Nonetheless, that goal seems too far-fetched in the developing world where there are hundreds of villages, towns and cities without adequate infrastructures. Perhaps the key to solving this problem is combining point-of-use-purification of water (by chlorination) with the treatment of illness in the community. Treatment of chronic carriers is also necessary in order to halt the cycles of transmission.
\end{abstract}

Key Words: Enteric fever, typhoid fever, paratyphoid fever, antibiotic resistance, antibiotic recycling and nalidixic acid resistance

J Infect Developing Countries 2008; 2(6):426-430.

Received 27 June 2008 - Accepted 4 August 2008

Copyright $\odot 2008$ Arjyal and Pandit. This is an open access article distributed under the Creative Commons Attribution License, which permits unrestricted use, distribution, and reproduction in any medium, provided the original work is properly cited.

Enteric fever, the septicaemic illness caused by the bacteria Salmonella Typhi or Paratyphi A, is still perceived as a readily treatable illness by many clinical practitioners in those areas of the world, especially South and Southeast Asia, where the disease is highly endemic. Nevertheless, in recent years, the emergence of strains resistant to conventional treatment, the reports of treatment failures with trusted drugs, and the continued persistence of Typhi and emergence of Paratyphi A disease [1,2,3,4,5] all point to a need for a reappraisal of approaches to the treatment of enteric fever. A close examination of how the various treatments against this disease have evolved and are perceived gives valuable insights into the challenges posed by it and the opportunities to counter them.

The often fatal and severe illness of typhoid was first rendered treatable with the introduction of chloramphenicol in 1948 [6]. Although the first reports of resistance to chloramphenicol emerged within a few years [7], it was still used as the treatment of choice for enteric fever up until the early 1990s [8,9]. Besides treatment failures and the rise of resistance, the fear of chloramphenicol causing bone marrow depression has undoubtedly also had a role in treatment providers' choice of alternative first-line drugs such as ampicillin and cotrimoxazole. In the 1990s, a rise in resistance to all three first-line drugs [3] led physicians to move away from these drugs altogether.

The interval between the introduction of ciprofloxacin in the late $1980 \mathrm{~s}$ and its rise to prominence as the drug of choice for enteric fever was not long and the fluoroquinolones have been the mainstay of treatment for enteric fever since then. Ciprofloxacin was followed by another fluoroquinolone, ofloxacin, initially used in low doses and durations and then subsequently recommended to be used at higher doses in areas where resistance was expected [10,11]. This class of drugs is active against Gram-negative bacteria and very effectively kills intracellular Salmonella. This is due to a property of the class that allows them to be deposited intracellularly at a concentration that is up to ten times greater than that in the extracellular space $[12,13,14]$.

There have also been widespread reports of treatment failures with the fluoroquinolone class of drugs when used at conventional doses [15]. These 
treatment failures have been observed even when there was no resistance to these drugs shown from in vitro tests for sensitivity, using the currently recommended antimicrobial susceptibility breakpoints (CLSI, BSAC). This failure has been correlated with the phenomenon of resistance to the parent quinolone, nalidixic acid, widely prevalent in currently circulating strains. Nalidixic acid resistance is thus a marker for potential clinical resistance [16,17] to treatment with fluoroquinolones, even though the bacterium may be sensitive in vitro. However, this view has been challenged by the findings of recent papers $[4,18]$ which show that nalidixic acid resistant screening does not indentify all Salmonella Typhi isolates with decreased ciprofloxacin susceptibility and thus the clinical failures. A decreased ciprofloxacin susceptibility with MICs 0.12 to $1 \mathrm{microgram} / \mathrm{millilitre}$ seems to be a better predictor of poor clinical response.

There has been recognition that higher doses of the fluoroquinolones will have to be used to gain the same treatment effects as were originally seen [19]. This observation also suggests the emergence of resistance because, if resistance is a continuous spectrum, from full sensitivity to complete resistance, then the fact that a previously effective dosage of a drug is no longer successful carries the implication that resistant strains have already emerged.

There is a belief that if the use of fluoroquinolones could be stopped and alternatives such as azithromycin or the extended spectrum cephalosporins were used in areas with high rates of quinolone resistance, there might be a return to susceptible strains, akin to the phenomenon of chloramphenicol susceptibility reemergence. However, it can be hypothesised that this might not occur, as the chromosomal mutation that imparts resistance may not be a burden to the bacteria when it comes to survival, leading the resistant strains to persist.

The new fluoroquinolone, gatifloxacin, is another drug that has made it to the front line in the battle against enteric fever. Although a fluoroquinolone, it behaves slightly differently from the other fluoroquinolones in that it binds at a slightly different site [20], such that treatment failures due to resistance gained by point mutations of the older binding site can still be averted. Its usefulness has been proven in two trials where it was used with high clinical success rates in areas with high rates of nalidixic acid resistance [21,11].

Hugely successful at quickly clearing fever compared to their counterparts, fluoroquinolones were widely used in adults; however, reports of joint damage in experimental animals precluded the use of fluoroquinolones in children until prospective studies established that they are acceptable in children when alternatives are not available [22]. Even now, it has yet to be established conclusively beyond what age the fluoroquinolones can be used; whether they can be used in infants and very young infants; and whether they can be used safely in pregnancy. There is also some degree of hesitation when it comes to using the fluoroquinolones in the higher doses that would be required to counter resistant strains, albeit that these drugs have a wide therapeutic index and hardly any instance of fluoroquinolone poisoning has been chronicled.

Gatifloxacin has also caused much debate due to reports that it is associated with dysglycemia in the elderly or in diabetics. Dysglycemia in the elderly could very likely be due to the tendency of compromised renal function in the elderly, leading to a decreased clearance of the drug. This may not apply to a younger population, which is disproportionately afflicted by enteric fever as shown by the use of gatifloxacin in the treatment of other diseases in children [23]. Evidence from a case control study [24] showed a higher proportion of the elderly among cases compared to controls and remarkably high odds for the likelihood of dysglycemia. However, there is an equal chance that those of the elderly with severe infections, and thereby those with a greater chance of developing renal compromise or glucose homeostasis abnormalities, were administered the newer and more potent broad spectrum fluoroquinolone. A recent study comparing gatifloxacin with cefixime in the treatment of uncomplicated enteric fever showed gatifloxacin to be effective and well tolerated in a largely young and healthy Nepalese population [13]. It is now very important to perform a prospective study to determine the potential risk of dysglycemia in young healthy adults. Until then, the use of gatifloxacin should be guided by benefit versus risk.

The third generation cephalosporins are another class of drugs used to treat enteric fever. Intravenous formulations of these have been very useful for the treatment of bacterial septicaemia in general and a broad spectrum of activity is afforded by this class of drugs. Together, this means that their use is initiated whenever a patient with suspected but undifferentiated septicaemia is encountered. Additionally, there have been only a few reported instances $[25,26]$ of overt in vitro $S$. Typhi and Paratyphi resistance to this class of 
drugs. This fact and the recommendations [27] for use of this class of drugs as a second-line treatment against enteric fever means that clinicians use it when the other drugs fail, or when the illness is severe. The recommended doses of ceftriaxone and cefixime for treatment of uncomplicated enteric fever are $60 \mathrm{mg} / \mathrm{kg}$ body weight for 10 to 14 days and $20 \mathrm{mg} / \mathrm{kg} /$ day for 7 to 14 days respectively [27]. However, a recent clinical trial undertaken in Kathmandu [11], and one earlier in Vietnam [28], have demonstrated prolonged fever clearance time, higher clinical failure, and relapse with cefixime as compared with flouroquinolones.

Azithromycin is a new drug for the treatment of enteric fever. Its appreciable intracellular concentration and activity against both nalidixic acid resistant and multidrug resistant strains means that it can be usefully employed even in areas where both types of resistance are prevalent, as demonstrated in a recently conducted clinical trial [21]. The recommended dose of azithromycin is $8-10 \mathrm{mg} / \mathrm{kg} /$ day for 7 days [27].

In recent years there have been reports of bacteria being sensitive to chloramphenicol in areas where its use was previously stopped, due to the rise in the proportion of resistant strains and associated treatment failures [2]. Resistance to chloramphenicol and other first-line drugs such as ampicillin and co-trimoxazole is plasmid-mediated. It can be hypothesised that the removal of drug pressure means that carrying the plasmid incurs extra nutritional needs for the bacterium and a negative survival advantage, thus explaining the rise in proportion of susceptible strains.

This also points to the phenomenon of unintentional antibiotic cycling, preserving the older antibiotic. The concept of antibiotic cycling has been used quite successfully in hospital settings [29], with nosocomial infections in mind, to avert the disaster of running out of useful antimicrobials. Its use in a community setting, however, would be more complex because it would mean the coordination of a whole array of treatment providers right from the corner drug-shop to tertiary care hospitals at the top of the health-care pyramid. It would also require the setting up of adequate surveillance to spot, early on, the trends of rising resistance to the drugs currently being used and to confirm that the strains currently causing disease are sensitive to a drug that would be used.

Another interesting question that the treatment of enteric fever raises is that of the balance between adequate dosing and toxicity of a drug. It is appreciated that a high enough dose of an antibacterial must be used such that adequate tissue and plasma levels are attained to be able to kill all the organisms in question and minimise the emergence of resistance. Unfortunately, unfounded fear of toxicity often stands in the way of optimum dosing. Such fears have begun with the use of chloramphenicol. While it has been often observed that chloramphenicol causes reversible bone marrow suppression leading to leucopenia and thrombocytopenia [30], it is difficult to reliably establish causation in the cases where chloramphenicol has been purported to cause aplastic anemia in one among 24,000 to 40,000 cases that have been treated with it [31]. Although a valuable drug for the treatment of many infectious diseases besides enteric fever, and practically a lifesaver in many scenarios in the vast rural swathes of the developing world, the use of chloramphenicol is still perceived with much uncertainty.

The question of whether parenteral antimicrobials are superior to oral drugs is also an interesting one when it comes to enteric fever. The third-generation cephalosporins were initially only produced in parenteral formulations. But, given the demonstrated prolonged fever clearance time of the third-generation cephalosporins compared to the fluoroquinolones [19], the question must be asked whether a newer oral fluoroquinolone such as gatifloxacin or a parenteral cephalosporin such as ceftriaxone is of superior value, even in the treatment of a patient with severe illness. The fluoroquinolones have high levels of oral bioavailability and the difference between administering a parenteral drug of lesser potency and an oral drug of greater potency in a severely ill patient has not been clearly outlined.

Severe enteric fever also presents many challenges to treatment. Frequently, severe enteric fever is not identified with certainty in the first place because of the generally non-specific features of the illness and the absence of a specific and sensitive rapid diagnostic test. Also, blood cultures may not yield the organism after patients have been partially treated with antibacterials, as they most commonly are before they seek treatment for severe illness. Even when they do demonstrate the organism, at a level of sensitivity as low as 30\% (range $30-90 \%$ ) [32], blood cultures still leave a lot to be speculated upon, leaving only the option of empirical treatment. Empirical treatment for presumed bacterial sepsis is often undertaken in such critical situations. The choice of antibiotics is still a challenge to the clinicians working in developing countries where the aetiology of febrile illness includes typhoid, rickettsiosis, and leptospirosis [33]. The usefulness of steroids in severe 
typhoid was demonstrated by a single trial from Indonesia where the patients were treated with chloramphenicol with steroids [34]. Whether similar results will be obtained when steroids are used with ceftriaxone or a fluoroquinolone is a question yet to be answered.

In the industrialised world, incidences of suspected enteric fever which especially affect returning visitors are often greeted with alarm and subjected to combination antibiotic therapy [35]. There is evidence that at least one antibiotic combination-azithromycin and ofloxacin-appeared to confer no additional advantage to monotherapy with azithromycin [36] and it is not known whether combinations would affect carriage rates in the long term.

Another, often overlooked, aspect of the treatment of enteric fever is the supportive treatment. Because of the high pyrexia that the illness causes and the fact that even the most successful treatment takes a few days to alleviate the illness, generous antipyresis [37] plays an important role in alleviating symptoms. Alongside this, inadequate intake of fluids and nutrition, brought on by the extreme anorexia associated with the disease, often plays a major role in aggravating the severe symptoms.

Although, as a public health problem, enteric fever has been tackled by protection of food and water supplies in the industrialised countries of the world, that goal seems too far-fetched in the developing world where there are hundreds of villages, towns and cities without adequate infrastructures. Perhaps the key to solving this problem is combining point-of-use-purification of water by chlorination [38] with treatment of illness in the community. Detection and treatment of chronic carriers in order to halt the cycles of transmission is also a very important step. There might be a cue here from the successes of malaria control programmes which combined the use of bed-nets with treatment $[39,40]$. While larger infrastructures such as sewage and water supplies will take enormous amounts of resources to construct, point-of-use water purification is an achievable and very low-cost intervention.

Thus there are clearly only a handful of options for reliably treating enteric fever. It is important to have information about the susceptibility patterns of the circulating organisms at a particular location, in order to make an educated choice about treatment. Adequate dosing and duration of treatment will not only play a role in achieving clinical success, but also in preventing relapses and carriage which will eventually play a role in transmission. The medical community must draw a line between objective evidence and subjective emotions when making clinical decisions and choosing treatments, and weigh the benefits and risks against each other. A combination of the current evidence and point-of-use purification of water may eventually relegate enteric fever to the level of importance it actually deserves.

\section{Acknowledgements}

The authors acknowledge that some of the ideas expressed in this article are from conservations with Dr. Jeremy Farrar and Dr. Buddha Basnyat. We express our sincere gratitude towards them.

\section{References}

1. Tankhiwale SS, Agrawal G, Jalgaonkar SV (2003) An unusually high occurrence of Salmonella enterica serotype Paratyphi A in patients with enteric fever. Indian J Med Res 117: 10-2.

2. Woodward TE, Smadel JE, Ley HL Jr, Green R, Mankikar DS (1948) Preliminary report on the beneficial effect of chloromycetin in the treatment of typhoid fever. Ann Intern Med 29:131-4.

3. Maskey AP, Basnyat B, Thwaites GE, Campbell JI, Farrar JJ, Zimmerman MD (2008) Emerging trends in enteric fever in Nepal: 9124 cases confirmed by blood culture 1993-2003. Trans R Soc Trop Med Hyg 102: 91-5.

4. Rowe B, Threlfall EJ, Ward LR (1987). Does chloramphenicol remain the drug of choice for typhoid? Epidemiol Infect 98:379-83.

5. Acharya G, Butler T, Ho M, Sharma PR, Tiwari M, Adhikari RK et al. (1995) Treatment of typhoid fever: Randomized trial of a three-day course of ceftriaxone versus a fourteen-day course of chloramphenicol. Am J Trop Med Hyg 52:162-65.

6. Dolecek C, Phi La TTP, Rang NN, Phuong LT,Vinh H, Tuan PQ et al. (2008) A multi-center randomised controlled trial of gatifloxacin versus azithromycin for the treatment of uncomplicated typhoid fever in children and adults in Vietnam. PLoS One 3(5): e2188. doi:10.1371/journal.pone.0002188.

7. Kato Y, Fukayama M, Adachi T, Imamura A, Tsunoda T, Takayama N et al. (2007) Multidrug resistant typhoid fever outbreak in travellers returning from Bangladesh. Emerg Infect Dis 13:1954-5.

8. Parry CM, Ho VA, Phuong le T, Bay PV et al. (2007) Randomized controlled comparison of ofloxacin, azithromycin and an ofloxacin-azithromycin combination for the treatment of multidrug-resistant and nalidixic acid-resistant typhoid fever. Antimicrob Agents Chemother 51:819-25.

9. Bethell DB, Hien TT, Phi LT, Day NP, Vinh H (1996) Effects on growth of single short courses of fluoroquinolones. Arch Dis Child 74:44-6.

10. Yunis AA, Smith US, Restrepo A. Reversible bone marrow suppression from chloramphenicol (1970) A consequence of mitochondrial injury. Arch Intern Med 126(2) 272-5.

11. Wallerstein RO, Condit PK, Kasper CK, Brown JW, Morrison FR (1969) Statewide study of chloramphenicol therapy and aplastic anemia. JAMA 208(11) 2045-2050.

12. Vinh H, Wain J, Hanh VTN, Nga CN, Chinh MT, Bethell D et al. (1996) Two or three days of ofloxacin treatment for uncomplicated multidrug-resistant typhoid fever in children. Antimicrob Agents Chemother 40: 958-61.

13. Pandit A, Arjyal A, Day JN, Paudyal B, Dangol S, Zimmerman D et al. (2007) An open randomized comparison of gatifloxacin 
versus cefixime for the treatment of uncomplicated enteric fever. Plos One; 2 (6): e542. doi:10.1371/journal.pone.0000542.

14. Parry CM, Hien TT, Dougan G, White NJ, Farrar JJ (2002) Typhoid fever. N Engl J Med 347: 1770-82.

15. Park-Wyllie LY, Juurlink DN, Kopp A, Shah BR, Stukel TA et al (2006) Outpatient gatifloxacin therapy and dysglycemia in older adults. N Engl J Med 354:1352-61.

16. Hoffman SL, Punjabi NH, Kumala S et al. (1984). Reduction of mortality in chloramphenicol-treated severe typhoid fever by high-dose dexamethasone. N Engl J Med 310:82-8.

17. Wain J, Kidgell C (2004) The emergence of multidrug resistance to antimicrobial agents for the treatment of typhoid fever. Trans R Soc Trop Med Hyg 98: 423-30.

18. Crump JA, Kretsinger K, Gay K, Hoekstra RM, Vugia DJ, Hurd S et al. (2008) Clinical response and outcome of infection with Salmonella enterica serotype typhi with decreased susceptibility to fluoroquinolones: a United States foodnet multicenter retrospective cohort study. Antimicrob Agents Chemotherap 52:1278-84.

19. Chau TT, Campbell JI, Galindo CM, Hoang NVM, Diep TS, Nga TT et al. (2007) Antimicrobial drug resistance of Salmonella enterica serovar typhi in Asia and the molecular mechanism of reduced susceptibility to the fluoroquinolones. Antimicrob Agents Chemother 51:4315-23.

20. Rupali P, Abraham OC, Jesudason MV, John TJ, Zachariah A, Sivaram S et al. (2004) Treatment failure in typhoid fever with ciprofloxacin susceptible Salmonella enteric serotype Typhi. Diag Microbiol Infect Dis 49:1-3.

21. Pichichero ME, Arguedas A, Dagan R, Sher L, Saez-Llorens X, Hamed K et al. (2005) Safety and efficacy of gatifloxacin therapy for children with recurrent acute otitis media (AOM) and/or AOM treatment failure. Clin Infect Dis 41:470-8.

22. Mirza SH, Beeching NJ, Hart CA (1996) Multi-drug resistant typhoid: a global problem. J Med Microbiol 44:317-319.

23. Pascual A, Garcia I, Perea EJ (1989) Fluorometric measurement of ofloxacin uptake by human polymorphonuclear leukocytes. Antimicrobial Agents and Chemotherapy 33: 653-6.

24. Chateau MT, Caravano R (1993) Rapid Fluorometric measurement of the intracellular concentration of ciprofloxacin in mouse peritoneal macrophages. Journal of Antimicrobial Chemotherapy 31:281-7.

25. Taira K, Koga H, Kohno S (1993). Accumulation of a newly developed fluoroquinolone, OPC-17116, by human polymorphonuclear leukocytes. Antimicrobial Agents and Chemotherapy 37:1877-81.

26. Communicable Disease and Surveillance and Response Vaccines and Biologicals: World Health Organization (2003) Treatment of typhoid fever. Background document: the diagnosis, prevention and treatment of typhoid fever. Available:

www.int/entity/vaccine_research/documents/typhoid_diagnosis .pdf via the Internet.

27. Capoor MR, Nair D, Hasan AS, Aggarwal P, Gupta B (2006) Narrowing therapeutic options in typhoid fever, India. Southeast Asian J Trop Med Public Health 37: 1170-1174.

28. Saha SK, Talukder SY., Islam M, Saha S (1999) A highly ceftriaxone-resistant Salmonella typhi in Bangladesh. Pediatr Infect Dis J 18: 387.

29. Lu T, Zhao X, Dhrlica K (1999) Gatifloxacin activity against quinolone resistant gyrase: Allele specific enhancement of bacteriostatic bactericidal activities by the C-8 methoxy group. Antimicrob Agents Chemother 43: 2969-2974.

30. John JF (2000) In: Antibiotic cycling: is it ready for prime time? Infect Control Hosp Epidemiol 21: 9-11

31. Kadhiravan T, Wig N, Kapil A, Kabra SK, et al. (2005) Clinical outcomes in typhoid fever: adverse impact of infection with nalidixic acid-resistant Salmonella typhi. BMC Infect Dis 5:37.

32. Hakanen A, Kotilainen P, Jalava J, Siitonen A, Huovinen P (1999) Detection of decreased fluoroquinolone susceptibility in Salmonellas and validation of nalidixic acid screening test. J Clin Microbiol 37: 3572-3577.

33. Wain J, Diep TS, Ho VA, et al. (1998) Quantitation of bacteria in blood of typhoid fever patients and relationship between counts and clinical features, transmissibility, and antibiotic resistance. J Clin Microbiol 36:1683-1687.

34. Vinh H, Parry CM, Hanh VT, Chinh MT et al. (2004) Double blind comparison of ibuprofen and paracetamol for adjunctive treatment of uncomplicated typhoid fever. Pediatr Infect Dis J 23(3):226-30.

35. Nevill CG, Some ES, Mung'ala VO, Mutemi W, New L, Marsh K, Lengeler C, Snow RW (1996) Insecticide-treated bednets reduce mortality and severe morbidity from malaria among children on the Kenyan coast. Trop Med Int Health 1: 139-146.

36. Picard J, Aikins M, Alonso PL, Armstrong-Schellenberg JR, Greenwood BM, Mills A, 1993. A malaria control trial using insecticide-treated bed nets and targeted chemoprophylaxis in a rural area of The Gambia, West Africa. 8. Cost-effectiveness of bed net impregnation alone or combined with chemoprophylaxis in preventing mortality and morbidity from malaria in Gambian children. Trans R Soc Trop Med Hyg 87 (Suppl 2): 53-57.

37. Cao XT, Kneen R, Nguyen TA, Truong DL et al. (1999) A comparative study of ofloxacin and cefixime for treatment of typhoid fever in children. The Dong Nai Pediatric Center Typhoid Study Group. Pediatr Infect Dis J 18(3):245-8.

38. Cooke FJ, Day M, Wain J, Ward LR, Threlfall EJ (2007) Cases of typhoid fever imported into England, Scotland and Wales (2000-3) Trans R Soc Trop Med Hyg 101:398-404.

39. Murdoch D, Woods CW, Zimmerman MD, Dull PM, Belbase RH, Keenan AJ, Scott RM, Basnyat Archibald BLK, Reller LB (2004) The etiology of febrile illness in adults presenting to Patan hospital in Kathmandu, Nepal. Am J Trop Med Hyg 70:670-675.

40. Arnold BF, Colford JM (2007) Treating water with chlorine at point-of-use to improve water quality and reduce child diarrhea in developing countries: a systematic review and meta-analysis. Am J Trop Med Hyg 76(2):354-64.

Corresponding Author: Anil Pandit, Department of Internal Medicine, University of Utah Health Sciences Center, University of Utah, Salt Lake City, UT, USA, 84132 Email: anil.pandit@hotmail.com

Conflict of interest: No conflict of interest is declared. 